REV EXP MED

$2019 ; 5(1)$.

Enero - Marzo

\title{
Asociación entre desnutrición y evolución hospitalaria en pacien- tes menores de cinco años de un hospital del norte del Perú
}

\author{
Alexis Ormeño-Julca ${ }^{1 a}$, Jorge Sosa-Flores ${ }^{2 b}$, Carmen Zegarra-Hinostroza ${ }^{3 b}$
}

\section{RESUMEN}

Objetivo: determinar la asociación entre la desnutrición y la evolución hospitalaria en pacientes menores de cinco años hospitalizados en el Servicio de Pediatría del Hospital Regional Lambayeque. Material y Métodos: estudio observacional analítico, de tipo cohorte retrospectiva. Se incluyeron 255 pacientes divididos en dos grupos: Grupo de expuestos (85 desnutridos) y grupo de no expuestos (170 no desnutridos). Resultados: la desnutrición estuvo asociada significativamente a las infecciones nosocomiales $(p<0.05)$ y tanto el reingreso hospitalario a los 90 días como la mortalidad, fueron más frecuentes en los pacientes desnutridos en comparación a los no desnutridos. A pesar que el promedio de días de hospitalización fue mayor en los pacientes desnutridos (11,98 $\pm 11,76$ días) en comparación con los no desnutridos $(9,63 \pm 12,02$ días), no se obtuvo una asociación estadísticamente significativa entre la desnutrición y la estancia hospitalaria prolongada $(p=0.058)$. Conclusiones: Se concluye que, en pacientes pediátricos hospitalizados, el reingreso a los 90 días y la mortalidad, se presentaron más frecuentemente en pacientes desnutridos, en comparación con los no desnutridos y las infecciones intrahospitalarias tuvieron una asociación estadísticamente significativa con la desnutrición al ingreso. La estancia hospitalaria prolongada no tuvo una asociación estadísticamente significativa con la desnutrición y probablemente este influenciada por otros factores como la presencia de comorbilidades

Palabras clave: desnutrición infantil, hospitalización, readmisión del paciente, mortalidad, infección hospitalaria (DeCS-BIREME).

\section{Association between malnutrition and hospital evolution in pa- tients under five years of age in a hospital in northern Peru}

\section{ABSTRACT}

Objective. To explore the consumption of pyrazinamide as an indicator of the incidence of tuberculosis (TB) in La Libertad region during the 2013-2017 period. Material and Methods. It was a retrospective cross-sectional study. The information was collected from the database of the Integrated Supply System for Medicines and Medical-Surgical Supplies of the Ministerio de Salud, which was used to calculate the amount of daily dose defined per 100,000 inhabitants per day. Data of the incidence of TB in La Libertad, notified to the epidemiological surveillance system (ESS), were obtained from the Department of TB Prevention and Control of the Ministerio de Salud. The information was processed and the data obtained after applying the Pearson correlation test with $\alpha=5 \%$. Results. There was a statistically significant downward trend $(r=0.9170, p=0.02)$ of pyrazinamide consumption during the study. A significant correlation was found $(r=0.8411, p<0.05)$ between consumption of pyrazinamide and the incidence of TB. The incidence rate per 100,000 inhabitants estimated from the consumption of pyrazinamide from 2013 to 2017 was 89.1, 82.2, 77.8, 74.6 and 75.8 , respectively; on average per year, it was $30 \%$ higher than the reported to the ESS. Conclusions. The consumption of pyrazinamide could be an indicator of TB incidence in La Libertad region.

Keywords: infant malnutrition, prolonged hospitalization, readmission, mortality, nosocomial infection.

\footnotetext{
${ }^{1}$ Hospital Regional Lambayeque, Lambayeque, Perú.

${ }^{2}$ Hospital Almazor Aguinaga Asenjo ESSALUD, Lambayeque, Perú.

${ }^{3}$ Hospital Regional Docente Las Mercedes, Lambayeque, Perú.

${ }^{\text {a }}$ Médico gastroenterólogo pediatra. Magister en Medicina.

${ }^{\mathrm{b}}$ Médico pediatra. Doctor en Medicina.
} 


\section{INTRODUCCIÓN}

La Organización Mundial de la Salud (OMS), estima que a nivel mundial casi uno de cada 4 niños menores de 5 años (165 millones, o el $26 \%$ en el 2011) sufre de desnutrición crónica, y más de 29 millones sufren desnutrición aguda, de forma moderada o grave, con la prevalencia más alta en el sur de Asia donde aproximadamente uno de cada seis niños sufre desnutrición aguda moderada o severa. La desnutrición aumenta el riesgo de complicaciones y muerte en pacientes con infecciones comunes de la infancia como neumonía, diarrea, malaria, VIH/SIDA o sarampión, teniendo los pacientes con desnutrición aguda grave nueve veces más probabilidades de morir que los niños eutróficos ${ }^{(1)}$.

Desde los años 80, varios estudios en diferentes países, han reportado que la desnutrición aguda es una condición frecuente en los pacientes hospitalizados ${ }^{(2-5)}$, con una prevalencia de desnutrición entre 6,1\% y 32\% en Alemania, Brasil, Francia, Inglaterra, Turquía, Estados Unidos y España ${ }^{(6,7)}$.

La desnutrición durante la estancia hospitalaria constituye un serio problema de salud debido al aumento de la morbi-mortalidad, mayor riesgo de infecciones por alteración de la función inmunológica, cicatrización alterada, reducción de la función intestinal, pérdida de masa muscular, necesidad de asistencia respiratoria prolongada y mayor estancia hospitalaria con un mayor costo económico en la atención. Las causas estarían relacionadas, tanto con la enfermedad subyacente, como con los procedimientos diagnósticos y terapéuticos instaurados durante el curso clínico de la patología y a otros aspectos de la hospitalización (8).

El objetivo general del presente estudio fue determinar la asociación entre la desnutrición y la evolución hospitalaria en pacientes menores de 5 años hospitalizados en el Servicio de Pediatría del Hospital Regional Lambayeque (HRL). Se planteó además como objetivos específicos determinar la asociación entre la desnutrición infantil y la estancia hospitalaria prolongada, el reingreso hospitalario a los 90 días, las infecciones nosocomiales y la mortalidad intrahospitalaria.

\section{MATERIAL Y MÉTODOS}

\section{Tipo y diseño de la investigación}

El presente es un estudio analítico, observacional de tipo cohorte, retrospectivo

\section{Población y muestra}

El trabajo abarcó los pacientes menores de 5 años hospitalizados en el Servicio de Pediatría del HRL en el periodo comprendido entre enero del 2014 hasta diciembre del 2016. Del total de 984 pacientes hospitalizados durante el periodo de estudio, ingresaron en forma aleatoria 255 , conformándose dos grupos: a) Grupo desnutridos ( $n=85$ ), que incluyó pacientes con distintos grados de desnutrición al ingreso (aguda, crónica, crónica reagudizada y severa).

b) Grupo no desnutridos ( $n=270)$ : sin desnutrición al ingreso. Para establecer el diagnóstico nutricional se tuvo en cuenta el peso al ingreso en todos los pacientes y la longitud en decúbito en menores de dos añosk, y talla en aquellos de dos años a más; calculando el z score de peso para la talla y de la talla para la edad.

Para el cálculo de la muestra se usó el programa estadístico Epidat 3.1, teniendo en cuenta los datos obtenidos por Machado et al. (9) quienes reportaron una estancia hospitalaria prolongada en pacientes desnutridos del $29 \%$ y en pacientes no desnutridos del $14 \%$ y considerando dos pacientes no expuestos por cada expuesto, con un nivel de confianza del $95 \%$ y una potencia de $80 \%$. El proceso de muestreo fue por selección aleatoria simple.

Se excluyeron los pacientes menores de un mes de edad, aquellos con patología neurológica (parálisis cerebral infantil, distrofia muscular, hidrocefalia congénita), cardiaca (cardiopatías congénitas y adquiridas con insuficiencia cardiaca congestiva), neumológica (displasia broncopulmonar, fibrosis quística) y enfermedades genético-metabólicas, pacientes con sobrepeso u obesidad; y pacientes con antecedente de parto pre término ( $<37$ semanas de edad gestacional) con menos de un año al momento del estudio, por la necesidad de curvas específicas para la evaluación antropométrica.

\section{Técnicas e instrumentos de recolección de datos}

Las variables estudiadas fueron: estancia hospitalaria prolongada (mayor a nueve días según el estándar para un hospital Nivel III-1), reingreso a los 90 días, infecciones intrahospitalarias y mortalidad nosocomial.

Se elaboró una ficha de recolección de datos con las variables a ser investigadas. Los datos antropométricos, días de hospitalización, presencia y tipo de infección intrahospitalaria, así como la mortalidad fueron tomados de la historia clínica hospitalaria de los pacientes.

Como indicadores del estado nutricional se utilizó el z score de peso para la talla $(P / T)$ y de talla para edad $(T / E)$, según criterios de la OMS, considerando a los pacientes como no desnutridos si el z score de ambos parámetros se encontraban entre $-2 y+2$ y como pacientes desnutridos aquellos con un $z$ score por debajo de -2 .

\section{Análisis de datos}

Para el análisis estadístico se calculó el Riesgo Relativo (RR) usando el programa Epidat 3.1 y para establecer la relación entre las variables analizadas se usó el programa 
SPSS $₫ 24.0$ para Windows, utilizando la Prueba de Chi2, con un nivel de confianza del 95\% considerando una asociación estadísticamente significativa si $p<0,05$.

\section{Consideraciones éticas}

Se contó con la aprobación tanto del Comité de Ética en Investigación como de la Dirección de Investigación del HRL, garantizando la confidencialidad de los datos obtenidos, en concordancia con los estándares éticos que rigen la investigación en seres humanos ${ }^{(10)}$.

\section{RESULTADOS}

Durante el periodo de estudio se hospitalizaron 984 pacientes, de los cuales 68 no fueron considerados por no tener datos completos en la historia clínica. Se excluyeron 183 pacientes, según los criterios establecidos, quedando 733 pacientes (642 eutróficos y 90 desnutridos). Luego del muestreo por selección aleatoria simple, finalmente se incluyeron 255 pacientes, 85 de ellos con desnutrición (grupo de expuestos) y 170 no desnutridos (grupo de no expuestos).

El promedio de días de hospitalización fue de 9,63 \pm 12,02 días para los pacientes no desnutridos y de 11,98 $\pm 11,76$ días para los desnutridos, sin embargo no se encontró asociación entre la desnutrición y la estancia hospitalaria prolongada. $R R=1.42$ (IC95\%:0,99-2,00; p>0,05). Cuatro pacien tes $(1,6 \%)$ fueron admitidos nuevamente en el Servicio de Pediatría dentro de los 90 días posteriores al alta, encontrando una asociación entre la desnutrición y el reingreso hospitalario a los 90 días $(p<0,05)$. Ver tabla 1.

Durante el período de estudio fallecieron tres pacientes $(1,2 \%)$, de los cuales todos eran desnutridos y dos de ellos tenían desnutrición severa. Se encontró una asociación entre la desnutrición y la mortalidad hospitalaria $(p<0,05)$. Sin embargo, tanto para el caso de reingreso hospitalario a los 90 días y mortalidad nosocomial, no fue posible calcular el RR debido a que la totalidad de eventos se presentaron en el grupo de expuestos (desnutridos).

Trece pacientes $(5,1 \%)$ presentaron durante la hospitalización infecciones nosocomiales. De estas la más frecuente fue neumonía intrahospitalaria $(69,2 \%)$, seguida por infección del tracto urinario $(23 \%)$ e infección del torrente sanguíneo $(7,8 \%)$. Se encontró una asociación entre la desnutrición y la presencia de infecciones nosocomiales en pacientes menores de cinco años con un $R R=4,5$ (IC95\%:1,42-14,19; $p<0,05)$. Ver tabla 1.

El 38,5 \% de los pacientes menores de cinco años con infecciones nosocomiales tuvo desnutrición crónica, el 7,7 $\%$ desnutrición aguda y el 23,1 \% desnutrición severa.

Tabla 1. Asociación entre el estado nutricional y evolución hospitalaria en menores de cinco años hospitalizados en el Servicio de Pediatría del Hospital Regional Lambayeque 2014-2016.

\begin{tabular}{|c|c|c|c|c|c|c|}
\hline \multirow[t]{2}{*}{ Variables } & \multicolumn{2}{|c|}{ Desnutrido } & \multicolumn{2}{|c|}{ No desnutrido } & \multirow[t]{2}{*}{$\begin{array}{l}\text { RR } \\
\text { (IC95\%) }\end{array}$} & \multirow[t]{2}{*}{ Valor $\mathrm{P}$} \\
\hline & $\mathbf{N}$ & $\%$ & $\mathbf{N}$ & $\%$ & & \\
\hline Estancia Prolongada & 34 & 13,3 & 48 & 18,8 & $\begin{array}{l}1.42 \\
(0,99- \\
2,01)\end{array}$ & 0,058 \\
\hline Reingreso Hospitalario a 90 días & 4 & $1,6 \%$ & 0 & 0,0 & - & $<0,05^{*}$ \\
\hline Mortalidad & 3 & $1,2 \%$ & 0 & 0,0 & - & $<0,05^{*}$ \\
\hline Infecciones nosocomiales & 9 & 3,5 & 4 & 1,6 & $\begin{array}{l}4.5(1,42- \\
14,10)\end{array}$ & $<0,05^{*}$ \\
\hline
\end{tabular}

$\left(^{*}\right)$ valor estadísticamente significativo (Prueba Chi2) 


\section{DISCUSION}

La desnutrición hospitalaria en niños, se reporta cada vez con mayor frecuencia por diferentes autores, siendo la desnutrición crónica la más frecuentemente encontrada ${ }^{(11)}$. Esto se debe a un inadecuado manejo nutricional con un impacto negativo en el crecimiento, que ya está establecido desde antes de la hospitalización, la falta de evaluación antropométrica rutinaria, que es un procedimiento sencillo, económico y no invasivo y a la ausencia de planes de Educación Nutricional efectivos en el primer nivel de atención (12,13).

La estancia prolongada es un indicador hospitalario cuestionado por estar influenciado por múltiples factores no siempre relacionados al aspecto nutricional, pero que aumentan el riesgo de infecciones, complicaciones digestivas y falla orgánica en pacientes desnutridos tal como lo reporta Simoes et al ${ }^{(11,14)}$, quienes encontraron que el tiempo de estancia hospitalaria posterior a un procedimiento quirúrgico era diferente, según la especialidad, siendo mayor en pacientes sometidos a trasplante hepático o portadores de alguna hepatopatía (media de 17,1 días) en comparación con aquellos sometidos a cirugía cardiaca, cirugía general, otorrinolaringológica, oftalmológica o plástica. A diferencia de lo reportado por varios autores ${ }^{(9,11,14-17)}$, en el presente estudio no se halló asociación estadísticamente significativa entre la desnutrición y la estancia hospitalaria prolongada, como también lo reportan Tarlovsky et al. ${ }^{(18)}$ y Velandia et al. ${ }^{(19)}$.

Esto puede explicarse debido a que se excluyeron pacientes con comorbilidad (patología neurológica, cardiaca, neumológica o con enfermedades genético-metabólicas), sobrepeso y obesidad, por estar asociadas por sí mismas a alto riesgo de morbimortalidad nosocomial, independientemente del estado nutricional, pudiendo condicionar una mayor estancia hospitalaria.

Sin embargo, si hubo un mayor promedio de días de estancia hospitalaria en pacientes con desnutrición en comparación con los eutróficos (11,98 vs 9,63 días). Asimismo, si consideramos solamente a los pacientes desnutridos, casi la cuarta parte de los pacientes con estancia hospitalaria prolongada tuvieron infección nosocomial y cerca del 12\% reingresaron al Hospital dentro de los 90 días posteriores al alta, lo cual muestra la influencia de la estancia hospitalaria prolongada sobre las infecciones nosocomiales y la readmisión a los servicios de hospitalización ${ }^{(19)}$.

En el caso del reingreso hospitalario, Lin et al, reporto en adultos, que los pacientes desnutridos tuvieron más riesgo de readmisión hospitalaria a los 15 días $(R R=1,9$; IC95\%: $1,1-3,2 ; p=0.025)$, a los 90 días ( $R R=1,4$; IC95\%: 0,9$2,0 ; p=0,101)$, y a los 6 meses ( $R R=1,3$; IC95\%: 1,0-1,8; $p=0,097$ ) en comparación con los pacientes eutróficos ${ }^{(20)}$. El único reporte en población pediátrica es el de Russell et al, quien estudio a 55 niños indígenas hospitalizados en Australia de 0,6 a 41,4 meses de edad, de los cuales en el $37 \%$ de pacientes la readmisión ocurrió dentro de los tres meses y en el $52 \%$ dentro de los seis meses posteriores al alta. Además, el $25 \%$ de todas las readmisiones fueron por diagnóstico primario de desnutrición (21)

En nuestro estudio, si bien se encontró asociación estadísticamente significativa entre la desnutrición y el reingreso a los 90 días, la totalidad de casos se presentó en el grupo de desnutridos, haciendo imposible la comparación de los riesgos tanto en el grupo de expuestos como en el de no expuestos. La inanición, secundaria a la ingesta disminuida de alimentos o por falta de apetito, condiciona un estado hipercatabólico, lo cual disminuye las reservas de energía en el tejido muscular y adiposo provocando déficit nutricional y complicaciones tanto en enfermedades agudas como crónicas, aumentado el riesgo de necesidad de hospitalización y por ende, de reingresos ${ }^{(22,23)}$.

La influencia del estado nutricional sobre la mortalidad infantil se ha reportado en varios estudios en los últimos años ${ }^{(24-26)}$. Al igual que Ngiravega ${ }^{(24)}$ en el presente estudio se encontró una asociación estadísticamente significativa entre la desnutrición (principalmente desnutrición severa) y la mortalidad hospitalaria, sin embargo al igual que en el caso de reingreso hospitalario a los 90 días, la totalidad de eventos se presentaron en el grupo de desnutridos.

La mortalidad y la desnutrición tienen una asociación no causal con las infecciones como causa básica de muerte, no siendo posible muchas veces establecer si la desnutrición fue causa antecedente o efecto en el episodio que finalmente conllevo a la muerte. Sin embargo si se puede afirmar que constituye el resultado de la interacción infección-desnutrición-infección ${ }^{(27)}$, como se puede observar en nuestra serie donde los tres pacientes fallecidos fueron desnutridos y dos de ellos tuvieron además infecciones intrahospitalarias.

En este estudio, al igual que Secker et al (28), también se encontró una asociación estadísticamente significativa entre la desnutrición y las infecciones nosocomiales. Quiroga reporta que la frecuencia de la enfermedad infecciosa como causa básica de muerte en menores de cinco años colombianos, es siete veces mayor cuando coexiste la desnutrición como causa antecedente ${ }^{(29)}$, lo cual demuestra la importancia de la interacción entre desnutrición e infección como binomio patológico de consecuencias extremas, particularmente en niños con otras enfermedades, como es el caso de los hospitalizados.

Se sabe además que la desnutrición es una de las causas más frecuentes de inmunosupresión, ya que puede afectar prácticamente todos los componentes del sistema inmune, pero principalmente al sistema inmune celular, condicionando linfopenia y alteración del cociente CD4/CD8.

Podemos concluir que en pacientes pediátricos hospitaliza 
dos, el reingreso a los 90 días y la mortalidad, se presentaron más frecuentemente en pacientes desnutridos, en comparación con no desnutridos. Las infecciones intrahospitalarias tuvieron una asociación estadísticamente significativa con la desnutrición al ingreso y la estancia hospitalaria prolongada no tuvo una asociación estadísticamente significativa con la desnutrición y probablemente este influenciada por otros factores como la presencia de comorbilidades.La limitación principal del presente estudio fue la poca cantidad de casos que presentaron los eventos estudiados dentro de la evolución hospitalaria.

Se recomienda realizar estudios prospectivos con una mayor muestra para establecer adecuadamente la asociación estadísticamente significativa entre la desnutrición infantil y la evolución hospitalaria en nuestra población.

Fuentes de financiamiento: Financiado por los autores.

Conflicto de Intereses: Ninguno.

\section{REFERENCIAS BIBLIOGRÁFICAS}

1.UNICEF Improving Child Nutrition: The achievable imperative for glo bal progress. 2013.

2.Marino LV, Goddard E, Workman L. Determining the prevalence of malnutrition in hospitalized paediatric patients. S Afr Med J. 2006; 96(9 $\mathrm{Pt})$ :993-5.

3.Sermet-Gaudelus I, Poisson-Salomon A-S, Colomb V, Brusset MC, Mosser F, Berrier F, et al. Simple pediatric nutritional risk score to identify children at risk of malnutrition. Am J Clin Nutr. 2000; 72(1):64-70.

4.De Moraes Silveira CR, De Mello ED, Antonacci Carvalho PR. Evolution of nutritional status of pediatric in patients of a tertiary care general hospital in Brazil. Nutr Hosp. 2008; 23(6):599-606.

5.Sarni RO, Carvalho MF, Monte CM, Albuquerque ZP, Souza FL. Anthropometric evaluation, risk factors for malnutrition, and nutritional therapy for children in teaching hospitals in Brazil. J Pediatr (Rio J). 2009; 85(3):223-8.

6.Joosten KF, Hulst JM. Prevalence of malnutrition in pediatric hospital patients. Curr Opin Pediatr. 2008; 20(5):590-6.

7.Lama More RA, Moráis López A, Herrero Álvarez M, Caraballo Chicano S, Galera Martínez R, López Ruzafa E et al. Validación de una herramienta de cribado nutricional para pacientes pediátricos hospitalizados. Nutr Hosp. 2012; 27(5):1429-36.

8.Beer S, Juarez M, Vega M, Canada N. Pediatric Malnutrition. Nutrition in Clinical Practice. 2015; 30(5):609-624.

9.Machado M, Ferreira M, Olivera R, Russo M, Babic B, Gimenez V, et al. Valoración antropométrica de los niños internados en el Centro Hospitalario Pereira Rosell. Arch Pediatr Urug. 2006; 77(2):110-117.

10 Asociación Médica Mundial; 64ª Asamblea General, Fortaleza, Brasil, octubre 2013. Declaración de Helsinki de la AMM-Principios éticos para las investigaciones médicas en seres humanos [En línea]. Fortaleza (Brasil): 2013. [Fecha de acceso 16 de Julio del 201]. URL disponible en: http://www.wma.net/

11. Hecht C, Weber M, Grote V, et al. Disease associated malnutrition correlates with length of hospital stay in children. Clin Nutr. 2015 Feb; 34(1):53-9.

12.Sullivan PB. Malnutrition in hospitalized children. Arch Dis Child. 2010;95:489-90.

13. Gomilla A, de Grandis E, Visconti G, et al. Estado nutricional en niños internados en salas de cuidados mínimos. Hospital de Niños de la Santísima Trinidad, 107. Córdoba: Arch Argent Pediatr; 2009; 107(1):37-42.

14.Simoes A, Zanin C, Vega R, Ferreira J, Oliveira F. Estado nutricional de crianças e adolescentes hospitalizados em enfermaria de cirurgia pediátri

ca. Rev Paul Pediatr. 2010;28(1):41-7.
15. Oropeza M, Rivera J, Gonzales J, Florian A, Rojas R. Evaluación Nutricional de niños hospitalizados con patología Gastrointestinal 2014-2015 en un Hospital pediátrico de tercer nivel. Lima, Perú. Resumen presentado en el 20 Congreso Latinoamericano y 11 Congreso Iberoamericano de Gastroenterología, Hepatología y Nutrición Pediátrica. 3 Congreso Peruano de Gastroenterología Pediátrica y Nutrición. 18-21 de Noviembre del 2015. Lima Perú. Rev Gastroenterol Peru 2015; 35(4):367-424.

16.Moeeni V, Walls T, Day A. Assesment of nutritional status and nutritional risk in hospitalized Iranian children. Acta Paediatrica. 2012; 101:e446-e451.

17.Moeeni V, Walls T, Day A. Nutritional Status and nutrition risk screening in hospitalized children in New Zealand. Acta Paediatrica. 2013; 102:e419-e423.

18. Tarlovsky V, Salmean G. Nutritional situation of hopitalized pediatric patients in a public hospital in Mexico City. Rev.Bras.Nutric.Clin. 2008; 23(3):178-183

19.Velandia S, Hodgson M, Le Roy C. Evaluación nutricional en niños hospitalizados en un Servicio de Pediatría. Rev Chil Pediatr. 2016; 87(5):359-365

20.Lim SL, Ong KC, Chan YH, Loke WC, Ferguson M, Daniels L. Malnutrition and its impact on cost of hiospitalization, length of stay, readmission and 3-year mortality. Clinical Nutrition. 2012; 31(3):345-50.

21.Russell B, White A, Newbury J, Hattch C, Thurley J and Chang A. Aust. J. Rural Health. 2004;12:187-191.

22. White J, et al. ASPEN / AND characteristics recommended for the identification and documentation of adult malnutrition. J Acad Nutr Diet. 2012;112:2012.

23.Jensen GL, Mirtallo J, Compher C, Dhaliwal R, Forbes A, Grijalba RF, et al. Adult starvation and disease-related malnutrition: A proposal for etiology-based diagnosis in the clinical practice setting from the International Consensus Guideline Committee. Clin Nutr. 2010; 34(2):156-9.

24.Ngirabega J, Munyanshongore C, Donnen P, Dramaix M. Influence of malnutrition on childhood mortality in a rural hospital in Rwanda. Revue d'Epidemiologie et de Sante Publique. 2011; 59:313-318.

25.Gabriele A, Schettino F. Child Malnutrition and mortality in developing countries: evidence from a cross-country analysis. 2007. Disponible sur: http://mpra.ub.uni-muenchen.de/3132/1/MPRA_paper_3132.pdf.

26.Babakissa C, Lacote-Povic S, Colombani J-F, Balan J-G. Risque nutritionnel en milieu hospitalier. Cahier Nutr Diet. 2004; 39(1):49-54.

27. Organización para la Agricultura y la Alimentación - FAO. Nutrición humana en el mundo en desarrollo. En: Nutrición e infección, salud y enfermedad. 2000. Fecha de consulta: 13 de abril de 2012. Disponible en: $\mathrm{ftp}: / / \mathrm{ftp}$.fao.org/docrep/fao/005/w0073s/W0073S00.pdf.

28.Secker DJ, Jeejeebhoy KN. Subjective global nutritional assessment for children. Am J Clin Nutr. 2007;85(4):1083e9.

29.Quiroga E. Mortalidad por desnutrición en menores de cinco años, Colombia, 2003-2007. Biomedica. 2012; 32:499-509.

Revisión de pares: Recibido: 04/02/2019 Aceptado: 21/03/2019 